\title{
A Novel Image Encryption Approach for Cloud Computing Applications
}

\author{
Saleh ALTOWAIJRI ${ }^{1}$ \\ Department of Information Systems \\ Faculty of Computing and \\ Information Technology \\ Northern Border University \\ Kingdom of Saudi Arabia
}

\author{
Mohamed AYARI ${ }^{2}$ \\ Department of Information \\ Technology \\ Faculty of Computing and \\ Information Technology \\ Northern Border University \\ Kingdom of Saudi Arabia
}

\author{
Yamen EL TOUATI ${ }^{3}$ \\ Department of Computer Science \\ Faculty of Computing and \\ Information Technology \\ Northern Border University \\ Kingdom of Saudi Arabia
}

\begin{abstract}
In this paper, a novel image encryption approach is proposed in the context of cloud computing applications. A fast special transform based on non-equispaced grid technique is introduced and applied as the first time in image encryption applications. By Combining with Fractional Fourier Transform (FRFT) instead of Discrete Fourier Transform (DFT), a good framework for image encryption is opened to enhance data security degree. The both image encipherment and decipherment process are analyzed based on random phase matrix. The time complexity effort of this novel approach is examined and evaluated. Comparative study with traditional encryption algorithms will prove the efficiency and robustness of our proposed technique.
\end{abstract}

Keywords-Cloud computing; image encryption; fourier transform; random phase function

\section{INTRODUCTION}

The tremendous advancement of information and communications technology (ICT) enables big documents to be effortlessly diffused online. Data encipherment is commonly applied to guarantee different security branches mainly data confidentiality, data integrity, and data availability. By the way, the majority algorithms used for enciphering are implemented for textual data. Despite these algorithms are efficient with this kind of data, they are too limited with multimedia applications by the reason of imposed constrains such as real time analysis and big data treatment. To confront these limitations, many algorithms have developed in the context of image encipherment and can be classified into two sets. The first one consists in transforming two-dimensional image matrix into unidimensional vector and then apply one of the well-known modern encryption techniques[1-3] like data encryption standard (DES), three-DES and advanced encryption standard (AES) belonging to symmetric key cryptosystems or RSA(Rivest-Shamir-Adelman) cipher, DiffieHellman key exchange and Elliptic curve cryptography (ECC) belonging to public-key cryptosystems (PKCS). The second one is to handle 2D- matrix presenting the authentic image and apply one of the available image encryption techniques [4-5] such as wave transmission [6] and two-dimensional discrete Fourier transform (2D-DFT).

In fact, wave transmission technique as its name indicates consists in replacing the value of each image pixel by self- adaptive wave. Taking into consideration the phase matrix keys, this method as well as chaotic system can be combined with DFT [7], fractional Fourier transform (FrFT) or any other image processing methods in order to improve the image encryption algorithms in terms of parallel implementation, speediness and multi-parameter selection.

FrFT is considered as a powerful tool in many sectors like signal processing, quantum mechanics and optics [8-10]. It is nothing but a generalization of the classical FT (Fourier Transform) characterized by the transform order which makes it suppler than FT [8-11], increases the key space and then improves the security degree of systems based on FrFT [1222].

The discrete version of FrFT so-called discrete fractional Fourier transform (DFrFT) can be obtained from DFT taking into consideration some free parameters [23-24]. In effect, the mathematical computation of DFrFT is achieved by the Eigen decomposition of the 2D-DFT matrix whereas the researchers have studied this decomposition in order to accelerate DfrFT algorithm by proposing different techniques to generate its eigenvectors such as commuting DFT matrix[25], HermitGaussian[23], direct batch evaluation[27] and singular value decomposition method[26].

In addition few years ago, random phase encoding has been applied in the image encryption process to elevate its security level [28]. It is introduced in fractional Fourier domain and combined with fractional order to offer an efficient key for enciphering/deciphering schemes. But, the noise-like aspect presenting in encrypted image becomes a deficiency since it opens a greedy gate for unauthorized user to make more and more attacks.

As mentioned above, the proposed solutions to improve data security in literature are concentrated on either the introduction of intelligent technique to reinforce the different transforms used in encryption scheme, the addition of specific key as random phase or the combination of them. Another research axis as presented in [8] can be much attractive for Scientists by rectifying the image encipherment process where the two Fourier transform operators have been replaced by two FRFT one. 
In this context, we propose a novel image encryption by introducing a new robust transform which is proposed for the first time in image encryption/decryption algorithms in order to ameliorate the security degree of data. This approach can be as well convenient to investigate cloud-based applications.

This paper is organized as follows: section II figures out the theoretical background and mathematical foundations building our novel image encryption process. Section III describes the proposed encryption/decryption algorithms. The analysis and evaluation of the proposed approach in the context of cloudbased applications are the subject of subsequent section (Section IV). Section V presents our conclusions and ongoing work.

\section{THEORETICAL BACKGROUND}

Many mathematical transformations are in the core of different disciplines related to image encryption and offer a good workspace to increase the data security. In this section, we will focus only on the aforementioned main important transformations that will be implemented and combined in the context of image encryption.

\section{A. Two Dimensional-Discrete Fourier Transform (2D-DFT)}

2D-DFT is considered as a very popular signal processing tool to understand the features of periodic, discrete-time signals in the frequency domain. Hence, it is very likely that it will be implemented in our proposed application.

The 2D-DFT of a sequence $\left(x_{k l}\right)$ can be defined as

$X_{m n}=\frac{1}{\sqrt{M * N}} \sum_{k=0}^{M-1} \sum_{l=0}^{N-1} x_{k l} * W_{N}^{n k} * W_{M}^{m l}$

So, its inverse can be written as:

$x_{k l}=\frac{1}{\sqrt{M * N}} \sum_{m=0}^{M-1} \sum_{n=0}^{N-1} X_{m n} * W_{N}^{-n k} * W_{M}^{-m l}$

Where

$W_{N}=e^{-j 2 \pi / N}$

$W_{M}=e^{-j 2 \pi / M}$

and $\left(x_{k l}\right)$ are a finite duration matrix with length $N^{*} M$.

\section{B. Fast Fractional Fourier Transform (FrFT)s}

Conventional Fourier transform takes the following expression:

$F(v)=(2 \pi)^{-\frac{1}{2}} \int_{-\infty}^{+\infty} f(t) e^{-j v t} d t$

$F(t)=(2 \pi)^{-\frac{1}{2}} \int_{-\infty}^{+\infty} F(v) e^{-j v t} d v$

Referred to (5) and (6), the $\alpha^{\text {th }}$ order fractional Fourier transform $F^{\propto}(v)$ can be written as follow:

$F^{\alpha}(v)=$

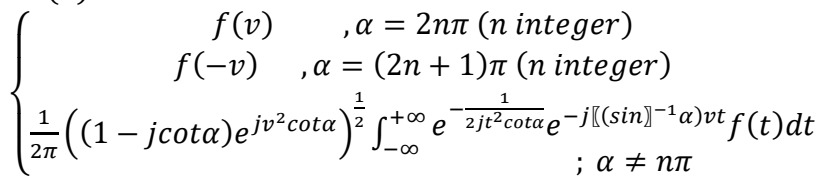

Obviously, FrFT has many properties like the conventional Fourier transform since FrFT is nothing but a general case of
FT. The reactions of $F^{\propto}(v)$ applied on a Pi function $\prod(t / 4)$ with low order $\alpha$ are given in Fig. 1. By the same way, the responses for high order are shown in Fig. 2.

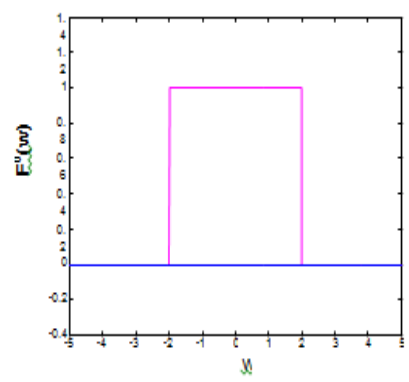

(a)

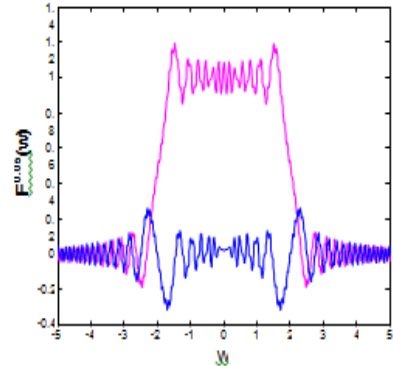

(c)

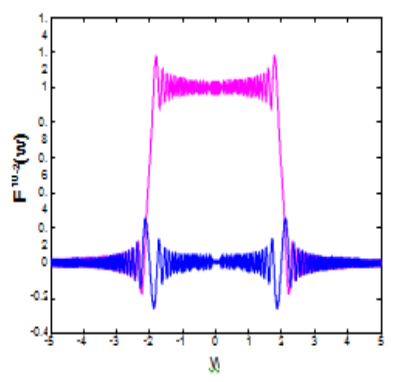

(b)

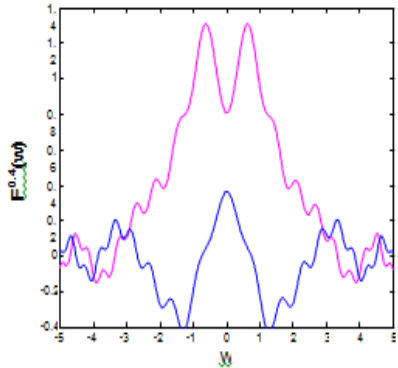

(d)
Fig. 1. FrFT with the order (a) $\alpha=0$ (b) $\alpha=1 / 100$ (c) $\alpha=1 / 20$ (d) $\alpha=2 / 5$ (Blue for imaginary part and pink for real part).

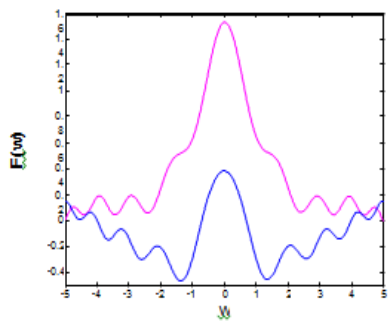

(a)

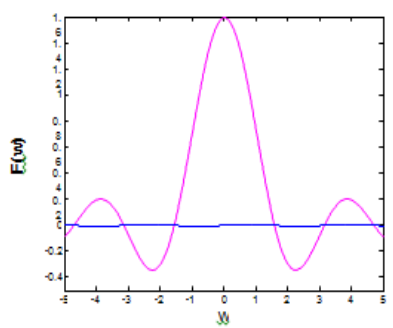

(c)

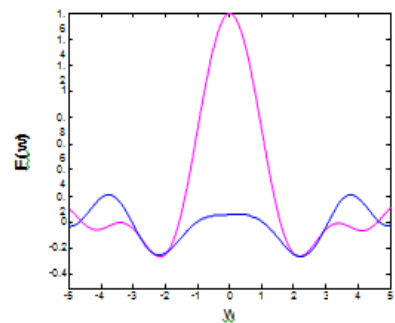

(b)

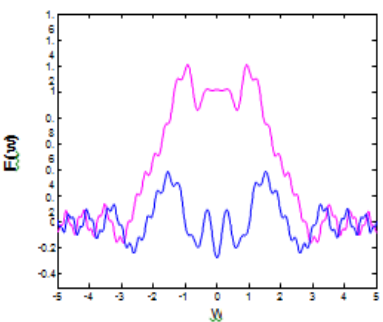

(d)
Fig. 2. FrFT with the order (a) $\alpha=\pi / 4$ (b) $\alpha=7 \pi / 16$ (c) $\alpha=\pi / 2$ (d) $\alpha=15 \pi / 16$ (Blue for imaginary part and pink for real part).

By optimizing the fundamental range of the fractional order, an efficient and rapid algorithm for numerical computation of FrFT has been investigated in [29] and refereed to [30], a two-dimensional FrFT based can be easily implemented in our work. 


\section{Two-Dimensional Fast Wavelet Transform (2D-FWT)}

$2 \mathrm{D}-\mathrm{FWT}$ is a numerically effective way of the 2D-discrete wavelet transform (2D-DWT) taking into account at adjacent scales the relationship between all 2D-DWT coefficients.

Let consider $\phi(x, y)$ a two-dimensional scaling function, and $\psi^{V}(x, y), \psi^{H}(x, y)$ and $\psi^{D}(x, y)$, three 2-D wavelets defined respectively as follows:

$\psi^{V}$ : Defines changes according to rows (i.e. vertical edges),

$\psi^{H}$ : Defines changes according to columns (i.e. horizontal edges),

$\psi^{D}$ : Defines changes according to diagonals,

Each of the aforementioned functions can be written as a multiplication of a unidimensional scaling function $\phi$ and its analogous wavelet $\psi$.

$\phi(x, y)=\phi(x) \phi(y)$

$\psi^{V}(x, y)=\phi(x) \psi(y)$

$\psi^{H}(x, y)=\psi(x) \phi(x)$

$\psi^{D}(x, y)=\psi(x) \psi(y)$

By applying numerical filters and its associated downsamplers, 2D-DWT can be easily implemented.

The diagram depicted in Fig.3 describes the twodimensional FT analysis filter bank. This one-scale filter bank can be repeated by applying the computation output to another filter bank input so as to generate as fractal scale system.

The following diagram as shown in Fig.4 presents two recurrences of the filtering method where the sub-images$W_{\phi}, W_{\psi}^{H}, W_{\psi}^{V}$, and $W_{\psi}^{D}$ are produced in the first iteration and the second one shows the two-scale decomposition.

To obtain the reverse problem already described in Fig. 3, the synthesis filter bank should be applied as depicted in Fig. 5:

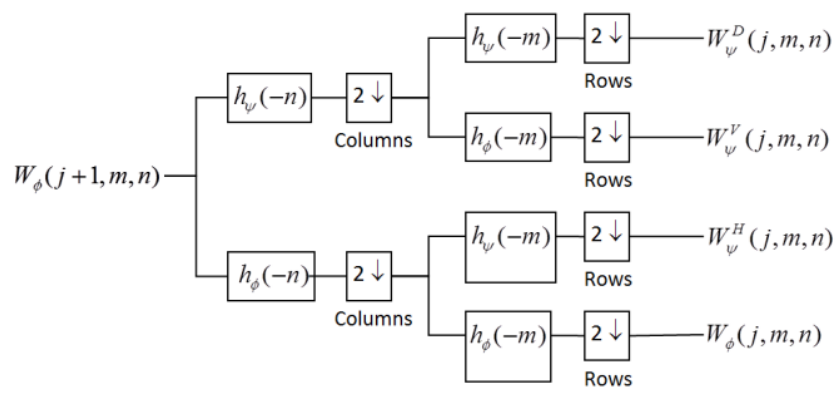

Fig. 3. 2D-FWT - Analysis Filter Bank.

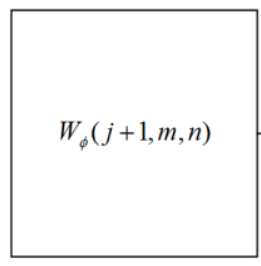

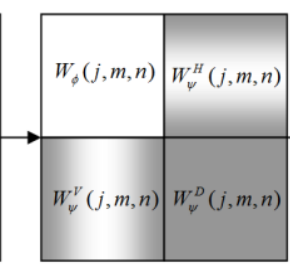

$1^{\text {st }}$ Iteration

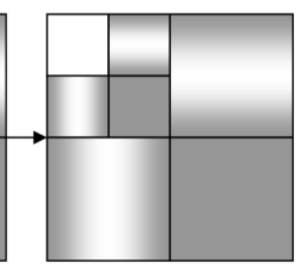

$2^{\text {nd }}$ Iteration
Fig. 4. Two-Scale of 2D-Decomposition.

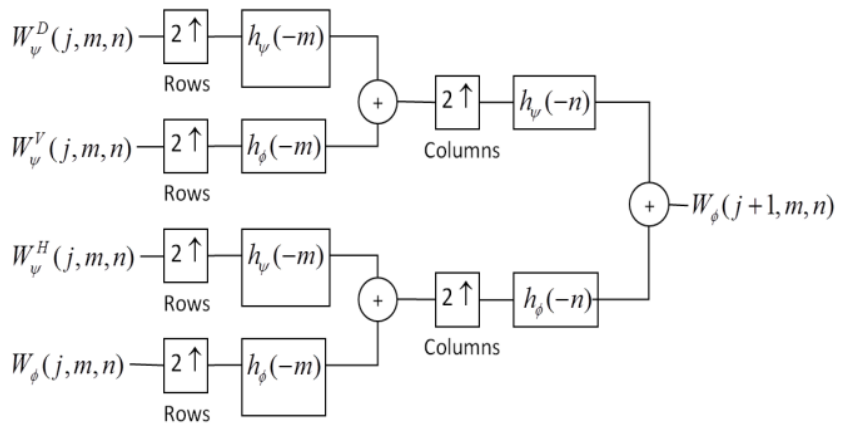

Fig. 5. 2D-IFWT - Synthesis Filter Bank.

\section{2D-Non-Uniform Fast Fourier Transform (2D-NUFFT)}

In image encryption applications, the 2D-NUFFT [31] developed by our research team- can be a good solution to enhance the security of data. But up to now, this technique has not introduced in the encryption domain. The non-uniformity and the speed of this mathematical transform can produce a powerful mechanism for many research applications. Under the frame of the numerical complexity, the conversion between spatial and Fourier domains is ensured at $O\left(N_{T} \log N_{T}\right)$ time ( $N_{T}$ is the total pixel numbers in $2 \mathrm{D}$ decomposition) while taking into consideration the non-equispaced of input data.

The efficiency of the 2D-NUFFT algorithm is equivalent to the FFT one. Therefore, this powerful transform will be implemented in this work by combining with aforementioned mathematical transformations. The schemes depicted in the following Figures (Fig.6 and Fig.7) [31] summarize this transformation and its inverse.

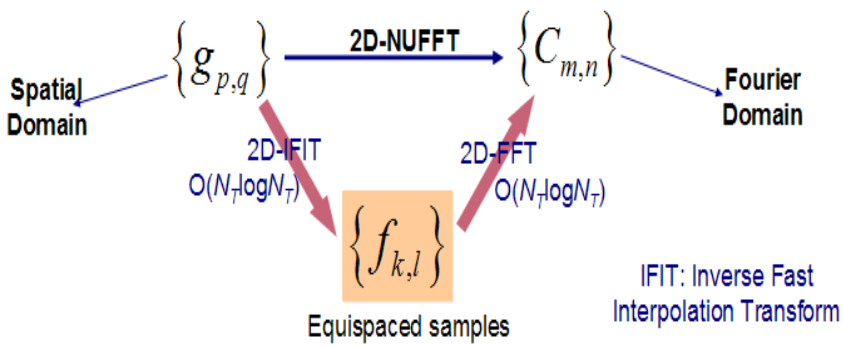

Fig. 6. 2D- NUFFT Scheme.

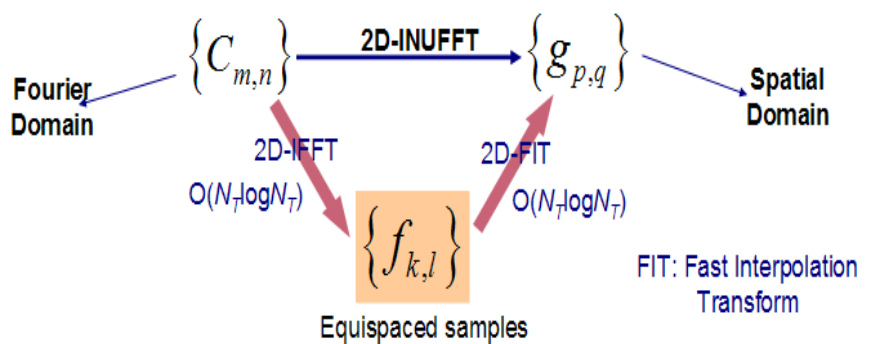

Fig. 7. 2D-Inverse NUFFT Scheme.

\section{PROPOSED ENCRYPTION/DECRYPTION ALGORITHMS}

In this section, we develop the new encryption and decryption schemes based on the different tools mentioned above in the context of image encryption applications. 
Let consider a 2D color image $I=F(x, y)$ (x and y are the spatial coordinates of the image) with size $M^{*} N$ identifying $R G B$ (Red, Green, Blue) channels.

This image can be represented as follows:

$I=\sum_{x=1}^{M} \sum_{y=1}^{N} F(x, y)$

Where

$F(x, y)=\sum_{1 \leq i \leq 3} f_{i}(x, y)$

Assume that these channels of the authentic image are separated. So, all different processes already described above will be applied simultaneously to RGB channels.

Initially, the $2 D-N U F F T$ transform is executed in each channel to produce:

$2 D-N U F F T\left\{f_{i}(x, y)\right\} \quad 1 \leq i \leq 3$

Then, the 2D-FWT is applied straightforwardly for each channel to generate:

$2 D-F W T\left\{2 D-N U F F T\left\{f_{i}(x, y)\right\}\right\} \quad 1 \leq i \leq 3$

The obtained distribution from (15) will be coded by the Chaotic Random Phase Mask (CRPM). This can be expressed arithmetically as follows:

$C R P M=e^{j \frac{\pi}{2} S(x, y)}$

$S(x, y)$ stands for the sequence with random number produced by the chaos function with selected seed value.

The 2-D FrFT transform is then implemented in this to produce:

$2 D-F r F T_{\alpha, \beta}\left\{2 D-F W T\left\{2 D-N U F F T\left\{f_{i}(x, y)\right\}\right\} *\right.$

$\left.e^{j \frac{\pi}{2} S(x, y)}\right\} \quad 1 \leq i \leq 3$

where $\alpha, \beta$ are 2D- FrFT fractional orders and * denotes the element-wise multiplication (i.e. Hadamard product) of equivalent matrices.

The inverse $2 D-F W T$ must be now applied at the resultant matrix obtained from (17).

In the final step, each channel will be operated with the inverse 2D-NUFFT to generate RGB channels associated to encoded image. This encrypted image $G(x, y)$ is generated after the combination of these three channels via the following equation:

$G(x, y)=\sum_{i=1}^{3} g_{i}(x, y)=$

$\sum_{i=1}^{3}\left\{2 D-I N U F F T\left\{2 D-I F W T\left\{2 D-F r F T_{\alpha, \beta}\{2 D-F W T\{2 D-N\right.\right.\right.$

$\left.\left.\left.\left.e^{j \frac{\pi}{2} S(x, y)}\right\}\right\}\right\}\right\}$

These steps can be seen in the following process (fig.8):

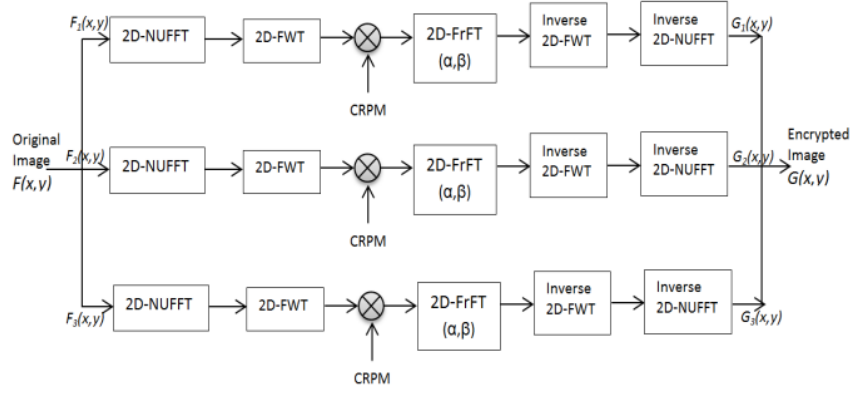

Fig. 8. Encryption Scheme in Proposed Algorithm.

The decipherment process given in the following figure (Fig.9) can be obtained from the reverse of the encipherment scheme as shown in Fig.8.

Indeed, the 2D-NUFFT operation should be applied to $G(x, y)$ for each channel in order to generate:

$2 D$-NUFFT $\left\{g_{i}(x, y)\right\} \quad 1 \leq i \leq 3$

Then, the 2D-FWT is directly applied on (19). It yields:

$2 D-F W T\left\{2 D-N U F F T\left\{g_{i}(x, y)\right\}\right\} \quad 1 \leq i \leq 3$

The inverse 2D- FrFT (with order $-\alpha,-\beta$ ) will be applied after that taking into consideration the CRPM conjugate already presented in equation (16).

We obtain:

$2 D-I F r F T_{-\alpha,-\beta}\left\{2 D-F W T\left\{2 D-N U F F T\left\{g_{i}(x, y)\right\}\right\} *\right.$

$\left.\operatorname{Conj}\left\{e^{j \frac{\pi}{2} S(x, y)}\right\}\right\} \quad 1 \leq i \leq 3$

Finally by applying the combination between inverse 2DFWT and inverse 2D-NUFFT on the outcome obtained in (21), the decrypted image can be now represented by the following distribution:

$F^{\prime(x, y)}=\sum_{i=1}^{3} f^{\prime}{ }_{i}(x, y)=$

$\sum_{i=1}^{3}\left\{2 D-I N U F F T\left\{2 D-I F W T\left\{2 D-I F r F T_{-\alpha,-\beta}\left\{2 D-F W T\left\{2 D-N U F F T\left\{f_{i}(x, y)\right\}\right\} *\right.\right.\right.\right.$

$\left.\left.\left.\left.\operatorname{Conj}\left\{e^{j \frac{\pi}{2} S(x, y)}\right\}\right\}\right\}\right\}\right\}$

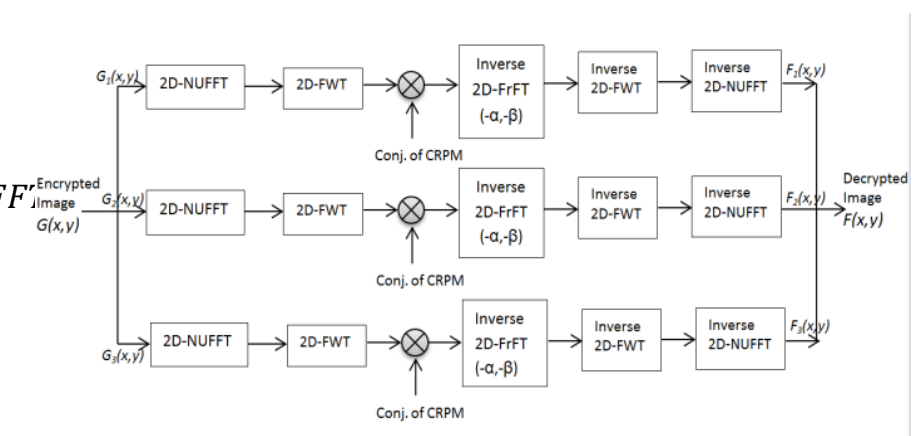

Fig. 9. Decryption Scheme in Proposed Algorithm. 


\section{ANALYSIS OF THE PROPOSED ALGORITHM}

The proposed algorithm is based on the combination between two powerful mathematical transforms - 2D-NUFFT and 2D-FWT - and also their inverses 2D-INUFFT and 2DIFWT in the same encryption or decryption process. The random phase function is produced as a 2-D sequence of random numbers and merged with the 2D-FrFT with order $(\alpha, \beta)$ in encryption process. By the way, the conjugate of the random phase function is used with the 2D-IFrFT with order ($\alpha,-\beta)$ in the decryption process.

In the following table, we compute the complexity of this algorithm in both encryption and decryption sides so as to evaluate the efficiency and robustness of our new image encryption scheme. We adopt $N^{*} N$ as the image size of $I$ in order to simplify the problem.

TABLE I. TIME COMPLEXITY OF THE PROPOSED ALGORITHM

\begin{tabular}{|c|c|c|c|}
\hline & Step & Description & Complexity \\
\hline \multirow{7}{*}{ 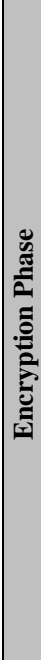 } & 1 & $\begin{array}{c}\text { Application of } 2 D-N U F F T \text { on the RGB } \\
\text { channels } f i(x, y)(1<=\mathrm{i}<=3) \text { of given authentic } \\
\text { image. }\end{array}$ & $O\left(N^{2} \log _{2} N\right)$ \\
\hline & 2 & Application of $2 D-F W T$ & $O(N)$ \\
\hline & 3 & Encoding by random phase function & $O\left(\frac{N^{2}}{2}\right)$ \\
\hline & 4 & Application of $2 D-F r F T$ with order $(\alpha, \beta)$ & $O\left(N^{3}+N \log _{2} N\right)$ \\
\hline & 5 & Application of $2 D-I F W T$ & $O(N)$ \\
\hline & 6 & $\begin{array}{c}\text { Application of } 2 D \text {-INUFFT on the RGB } \\
\text { channels. }\end{array}$ & $O\left(N^{2} \log _{2} N\right)$ \\
\hline & & Total time complexity of encryption & $O\left(N^{3}\right)$ \\
\hline \multirow{7}{*}{ 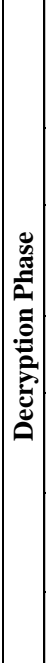 } & 1 & $\begin{array}{c}\text { Application of } 2 D-N U F F T \text { on the RGB } \\
\text { channels } f i(x, y)(1<=\mathrm{i}<=3) \text { of given authentic } \\
\text { image. }\end{array}$ & $O\left(N^{2} \log _{2} N\right)$ \\
\hline & 2 & Application of $2 D-I F W T$ & $O(N)$ \\
\hline & 3 & Encoding by conjugate random phase function & $O\left(\frac{N^{2}}{2}\right)$ \\
\hline & 4 & Application of $2 D-I F r F T$ with order $(-\alpha,-\beta)$ & $\mathrm{O}\left(\mathrm{N}^{3}+\mathrm{N} \log _{2} N\right)$ \\
\hline & 5 & Application of $2 D-I F W T$ & $O(N)$ \\
\hline & 6 & $\begin{array}{c}\text { Application of } 2 D \text {-INUFFT on the RGB } \\
\text { channels. }\end{array}$ & $O\left(N^{2} \log _{2} N\right)$ \\
\hline & & Total time complexity of decryption & $O\left(N^{3}\right)$ \\
\hline \multicolumn{3}{|r|}{ Total computation complexity of the system } & $O\left(N^{3}\right)$ \\
\hline
\end{tabular}

The evaluation of proposed encryption/decryption scheme by computing the number of its operations proves the speediness, efficiency and robustness of this algorithm in the context of image encryption applications. Furthermore in comparison with other methods developed in references [1620], the security degree becomes significant due to the introduction of the non-uniformity feature in the proposed process by the application of the NUFFT technique.

There is no doubt that cloud computing technology [32] is a developing sector of both computer and networking security and information security in large-scale

There are a set of significant strategy issues used to secure data, applications, and the related infrastructure of cloud-based resources, which include image encryption applications. Such proposed encryption/decryption algorithm shall be as accurately tailored as feasible to protect a big data and ensure a good workspace to handle with image having a high complexity problem.

\section{CONCLUSION}

In this paper, the theoretical background and mathematical foundations of the novel image encryption approach have been successfully developed and presented. The features of this approach as well as its strong points that set apart from other encryption schemes have been mentioned. Also, the detailed description of the proposed encryption and decryption process has been presented. The efficiency and robustness of our approach in terms of computational complexity have been demonstrated in the context of image encryption and cloud computing applications. The introduction of double chaotic random phase mask associated with $2 \mathrm{D}$ - FrFT in our approach can increase the security degree and circumvent the problem of big data met in cloud computing technologies.

\section{ACKNOWLEDGMENT}

The authors gratefully acknowledge the approval and the financial support of this research from the Deanship of Scientific Research study by the grant number CIT-2016-1-6F-5704, Northern Border University, Arar, KSA.

\section{REFERENCES}

[1] National Institute of Standards and Technology, "Data encryption standard (DES)," (1999).

[2] National Institute of Standards and Technology, "Advanced encryption standard (AES)," (2001).

[3] Rivest, R. L., Shamir, A., and Adleman, L., "A method for obtaining digital signatures and public-key cryptosystems," Communications of the ACM 21(2), 120-126 (1978).

[4] Zhou, Y., Panetta, K., Agaian, S., and Chen, C. L. P., "Image encryption using p-Fibonacci transform and decomposition," Optics Communications 285(5), 594-608 (2012).

[5] Zhou, Y., Panetta, K., Agaian, S., and Chen, C. L., “(n, k, p)-gray code for image systems," IEEE Trans Syst Man Cybern B Cybern (2012)

[6] Liao, X., Lai, S., and Zhou, Q., "A novel image encryption algorithm based on self-adaptive wave transmission," Signal Processing 90, 27142722 (2010).

[7] Chang, H. T., Hwang, H.-E., and Lee, C.-L., "Position multiplexing multiple-image encryption using cascaded phase-only masks in fresnel transform domain," Optics Communications 284(18), 4146-4151 (2011). 
[8] LIMA, Juliano B.; NOVAES, L. F. G. Image encryption based on the fractional Fourier transform over finite fields. Signal Processing, 2014, 94: 521-530.

[9] AWAL, Md Abdul, et al. A robust high-resolution time-frequency representation based on the local optimization of the short-time fractional Fourier transform. Digital Signal Processing, 2017, 70: 125144.

[10] Madrid, Y., Molina, M., \& Torres, R., "Quantum Fractional Fourier Transform. In Frontiers in Optics". Optical Society of America. (pp. JTu2A-73, September 2018.

[11] D. Mustard, "The fractional Fourier transform and the Wigner distribution," J. Aust. Math. Soc. B, vol. 38, pp. 209-219, 1996.

[12] R. Tao, B. Deng, and Y. Wang, "Research progress of the fractional Fourier transform in signal processing," Science in China (Ser.F, Information Science), vol. 49, pp. 1-25, Jan. 2006.

[13] G. Unnikrishnan and K. Singh, "Double random fractional Fourierdomain encoding for optical security," Opt. Eng., vol. 39, pp. 28532859, 2000.

[14] G. Unnikrishnan, J. Joseph, and K. Singh, "Optical encryption by double random phase encoding in the fractional Fourier domain," Opt. Lett., vol. 25, no. 12, pp. 887-889, 2000.

[15] Zhu B, Liu S, Ran Q: Optical image encryption based on multifractional Fourier transforms. Opt. Lett. 25 (2000),pp 1159-1161

[16] B. M. Hennelly and J. T. Sheridan, "Image encryption based on the fractional Fourier transform," Proc. SPIE, vol. 5202, pp. 76-87, 2003.

[17] R. Tao, Y. Xin, and Y. Wang, "Double image encryption based on random phase encoding in the fractional Fourier domain," Opt. Express, vol. 15, no. 24, pp. 16067-16079, 2007.

[18] R. Tao, X. M. Li, and Y.Wang, "Generalization of the fractional Hilbert transform," IEEE Signal Process. Lett., vol. 15, pp. 365-368, 2008.

[19] Hennelly B, Sheridan JT: Optical image encryption by random shifting in fractional Fourier domains. Opt. Lett. 28 (2003),pp 269-271.

[20] S. C. Pei and W. L. Hsue, "Random discrete fractional Fourier transform," IEEE Signal Process. Lett., vol. 16, no. 12, pp. 1015-1018, Dec.2009.

[21] L. J. Yan and J. S. Pan, "Generalized discrete fractional Hadamard transformation and its application on the image encryption," in Proc. Int.
Conf. Intelligent Information Hiding and Multimedia Signal Processing, 2007, pp. 457-460.

[22] H. Al-Qaheri, A. Mustafi, and S. Banerjee, "Digital watermarking using ant colony optimization in fractional Fourier domain," J. Inf. Hiding Multimedia Signal Process., vol. 1, no. 3, pp. 179-189, Jul. 2010.

[23] S. C. Pei and M. H. Yeh, "Improved discrete fractional Fourier transform," Opt. Lett., vol. 22, pp. 1047-1049, 1997.

[24] C. Candan, M. A. Kutay, and H. M. Ozaktas, "The discrete fractional Fourier transform," IEEE Trans. Signal Process., vol. 48, no. 5, pp. 1329-1337, May 2000.

[25] B. W. Dickinson and K. Steiglitz, "Eigenvectors and functions of the discrete Fourier transform," IEEE Trans. Acoust., Speech, Signal Process., vol. ASSP-30, pp. 25-31, Jan. 1982.

[26] M. T. Hanna, N. P. A. Seif, and W. A. E. M. Ahmed, "HermiteGaussian-Like eigenvectors of the discrete Fourier transform matrix based on the singular value decomposition of its orthogonal projection matrices," IEEE Trans. Circuits Syst. I, vol. 51, no. 11, pp. 2245-2254, 2004.

[27] M. T. Hanna, N. P. A. Seif, and W. A. E. M. Ahmed, "HermiteGaussian-Like eigenvectors of the discrete Fourier transform matrix based on the direct utilization of the orthogonal projection matrices on its Eigen-spaces," IEEE Trans. Signal Process., vol. 54, no. 7, pp. 2815 2819, Jul. 2006.

[28] Liu, Z., Chen, H., Blondel, W., Shen, Z., \& Liu, S. Image security based on iterative random phase encoding in expanded fractional Fourier transform domains. Optics and Lasers in Engineering, 105, 1-5, 2018.

[29] Yang, Xingpeng, Qiaofeng Tan, Xiaofeng Wei, Yong Xiang, Yingbai Yan, and Guofan Jin. "Improved fast fractional-Fourier-transform algorithm." JOSA A 21, no. 9 (2004): 1677-1681.

[30] Zayed, Ahmed. "Two-dimensional fractional Fourier transform and some of its properties." Integral Transforms and Special Functions (2018): 1-18.

[31] Ayari, Mohamed, Taoufik Aguili, and Henri Baudrand. "New version of TWA using two-dimensional non-uniform fast fourier mode transform (2d-nuffmt) for full-wave investigation of microwave integrated circuits." Progress In Electromagnetics Research 15 (2009): 375-400.

[32] Ali, Mazhar, Samee U. Khan, and Athanasios V. Vasilakos. "Security in cloud computing: Opportunities and challenges." Information sciences 305 (2015): 357-383. 